\title{
Lymphoscintigraphy in the Sentinel Lymph Node Technique for Breast Tumor: Value of Early and Late Images for the Learning Curve
}

\author{
C. Rousseau ${ }^{a}$ L. Campion ${ }^{b}$ C. Curteta J.M. Classe ${ }^{c}$ F. Dravet ${ }^{c}$ M. Fiche ${ }^{d}$ \\ C. Sagand J .F. Chatala I. Reschea
}

aNuclear Medicine, bStatistics, cSurgery, and dPathology Units, René Gauducheau Cancer Center, Nantes-Saint Herblain, France

\section{Key Words}

Breast cancer · Lymphatic mapping · Sentinel node

\begin{abstract}
As the performance of early $(\mathrm{H}+1$ to $\mathrm{H}+4)$ and late (D1) lymphoscintigraphic images raises organizational problems in outpatient surgery for breast cancer, only early images are generally obtained. The present study evaluated whether two series of images are better than one and defined the advantages of both methodologies. One hundred and eighteen patients with infiltrating breast carcinoma $\left(T_{0}, T_{1}\right.$ and $\left.T_{2}\right)$ were included in the study: 87 in group A (early and late images) and 31 in group $B$ (only early images). All patients received two peritumoral injections of 99mTc-sulfur colloid, 15-18 MBq (group A) and $<15 \mathrm{MBq}$ (group B). During the operation, the patent blue bye technique was associated with radioactivity detection. The two groups were comparable for histological type and tumor size and localization. Successful localization of sentinel nodes on early lymphoscintigraphic images was significantly greater for group $B$. The identification of a sentinel node focus on early lymphoscintigraphy increased by $10 \%$ during the study. Sentinel node detection by the isotopic method alone, or the two methods combined, was comparable for both groups. In radioactivity detection, the count rate for sen-
\end{abstract}

\section{KARGER}

Fax +41613061234

E-Mail karger@karger.ch

www.karger.com
(C) 2003 S. Karger AG, Basel

1011-7571/03/0121-0017\$19.50/0

Accessible online at:

www. karger.com/mpp tinel nodes versus background (contralateral breast) was similar for the two groups. During the learning phase, two series of images gave a definite advantage. Subsequently, lymphoscintigraphy performed at $+2 \mathrm{~h}$ was sufficient (the results for the two groups became indistinguishable).

Copyright @ 2003S. Karger AG, Basel

\section{Introduction}

Breast cancer screening has led to the treatment of an increasing number of subclinical cancers, but many axillary resections have proved negative because $75 \%$ of small tumors $(<2 \mathrm{~cm})$ are free of axillary metastases [1]. Nonetheless, the presence of an axillary node metastasis is a major prognostic factor in therapeutic strategy, and node status can only be determined by axillary resection and histopathological analysis of the nodes removed. Yet axillary resection, though essential to initial staging [2], can lead to postoperative morbidity, both immediately (lymphoceles) and subsequently (pain and lymphedemas). Given these circumstances, pre-and intraoperative sentinel node $(\mathrm{SN})$ detection would seem to be an attractive alternative.

The SN concept was introduced in 1977 by Cabanas [3] for cancer of the penis and then applied to the staging
C. Rousseau

Centre René Gauducheau

Service de Médecine Nucléaire, Boulevard Monod

F-44805 Saint Herblain Cedex (France)

Tel. +332 406799 31, Fax +332 406797 31, E-Mail c-rousseau@nantes.fnclcc.fr 
of melanoma, with detection by vital stains [4]. The excellent results obtained in these studies rapidly led to promising applications of these techniques to breast cancer, through injection of a labeled colloid and detection by an intraoperative probe $[5,6]$. With this approach, scintigraphic imaging is generally performed within $4-24 \mathrm{~h}$ after injection. If early images do not visualize the $\mathrm{SN}$, late images are recorded [7, 8]. However, the development of outpatient surgery, with the organizational constraints involved [9], has tended to limit imaging to a single series performed 1-2 $\mathrm{h}$ after injection of the radiopharmaceutical.

The purpose of this study was to determine whether two series of images are preferable to a single series and to define the advantages of the two methodologies.

\section{Materials and Methods}

One hundred and eighteen patients with infiltrating breast carcinoma $\left(\mathrm{T}_{0}, \mathrm{~T}_{1}\right.$ and $\left.\mathrm{T}_{2}\right)$ were included in the protocol from June 1999 to January 2001 . This prospective study was approved by the ethics committee in 1999. For 87 patients (group A), the radiopharmaceutical was injected on the day before surgery, and for the other 31 (group B) on the day of surgery. All patients received two injections of rhenium sulfide (Nanocis ${ }^{\circledR}$, Shering Cis Biointernational) performed peritumorally. Each syringe contained an activity of 15-18 MBq of technetium-treated Nanocis for group A and less than $15 \mathrm{MBq}$ (10.515 ) for group B. Injection volume for both groups was $0.1 \mathrm{ml}$. For group $\mathrm{A}$, images centered on the breast (included axilla, internal mammary and supraclavicular lymph nodes) were performed early $(\mathrm{H}+1$ to $\mathrm{H}+4)$ and late $(\mathrm{H}+20)$; for group $\mathrm{B}$, only early acquisition was performed just before surgery $(\mathrm{H}+1$ to $+1.5 \mathrm{~h})$. All images $(128$ $\times 128$ matrix) were in anterior incidence and in external profile of the breast studied; set imaging was $10 \mathrm{~min}$. No shielding was performed for the injection sites. In the operating room, 10-15 min before surgery, about $2 \mathrm{ml}$ of patent blue was injected peritumorally into all patients. The surgeon was guided by data from lymphoscintigraphy (LS). All surgeons were experienced in using detection probe. After tumor resection, the $\mathrm{SN}$ region was explored with a detection probe (Navigator, Tycon). Isolation of the $\mathrm{SN}$ for isotopic localization required a count rate equal to or twice that of the contralateral breast and/or the bluish appearance of a node rated at least ++ on a scale of 0 to +++ for the staining method. All patients underwent classical axillary resection (Berg stage I and II). Group assignment was not randomized, but based on whether a patient desired outpatient surgery (group B) or not (group A). In medical or surgical practice, a learning period is required before a new technique can be used routinely. All patients must be assured that results at least equivalent to those provided by the classical technique can be obtained in complete safety. In medical imaging, the same considerations apply for interpretation of a new examination, which requires a learning phase for optimal performance. During this period, the incidences used are not those adopted at the end of the study by an experienced observer, but those intended to allow optimal interpretation for an inexperienced observer.
The two groups (A and B) were compared for qualitative variables by the $\chi^{2}$ test (or Fisher's test in case of a limited number of patients), and for quantitative variables by Student's $t$ test (or the Mann-Whitney test in case of a limited number of patients); $p<0.05$ was considered significant.

\section{Results}

Although the groups of patients were not randomized, they were comparable in age and in the histological type, size and localization of their tumors (table 1). A comparable proportion of patients had positive LS (74.7 and $77.4 \%, \mathrm{p}=\mathrm{NS}$ ). During probe detection in vivo in the operating room, the count rate of the $\mathrm{SN}$ versus background (controlateral breast) was similar in the two groups: median $5.2(1.2-110)$ for group A and 9.1 (0.92245) for group B.

The success rate for $\mathrm{SN}$ localization in early images was significantly higher for group B (a single series of images) than for group $\mathrm{A}(\mathrm{p}=0.03$, table 2$)$. No difference

Table 1. Study population

\begin{tabular}{lcc}
\hline Parameters & Group A & Group B \\
\hline Patients & 87 & 31 \\
Mean age, years & $57 \pm 10.4$ & $59.1 \pm 14.9$ \\
Mean tumor size, mm & $21.2 \pm 2.3$ & $17.9 \pm 2.6$ \\
\hline Histopathology 1 & & \\
$\quad$ Ductal & 67 & 23 \\
$\quad$ Lobular & 9 & 5 \\
Other & 11 & 3 \\
$\quad$ Positive lymphoscintigraphy (\%) & $65(74.7 \%)$ & $24(77.4 \%)$ \\
\hline Tumor localization & & \\
$\quad$ Upper external quadrant & 47 & 17 \\
$\quad$ Upper internal quadrant & 16 & 4 \\
$\quad$ Lower external quadrant & 11 & 4 \\
$\quad$ Lower internal quadrant & 11 & 5 \\
Central & 2 & 1 \\
\hline
\end{tabular}

1 Exclusively invasive component.

Table 2. Success rate for localization of the SN by lymphoscintigraphy

\begin{tabular}{lll}
\hline Images & Group A & Group B \\
\hline Early $(\mathrm{H}+1$ to $\mathrm{H}+4)$ & $48(55 \%)$ & $24(77.4 \%)$ \\
Late $(\mathrm{H}+20)$ & $62(71 \%)$ & not applicable
\end{tabular}


Fig. 1. A, B Early images at $\mathrm{H}+2$. C, D Late images at $\mathrm{H}+20$. Anterior view $(\mathbf{A}, \mathbf{C})$ and left lateral view (B, D). Visualization of injection points on the left breast and uptake for the left axillary SN (seen only on late images).
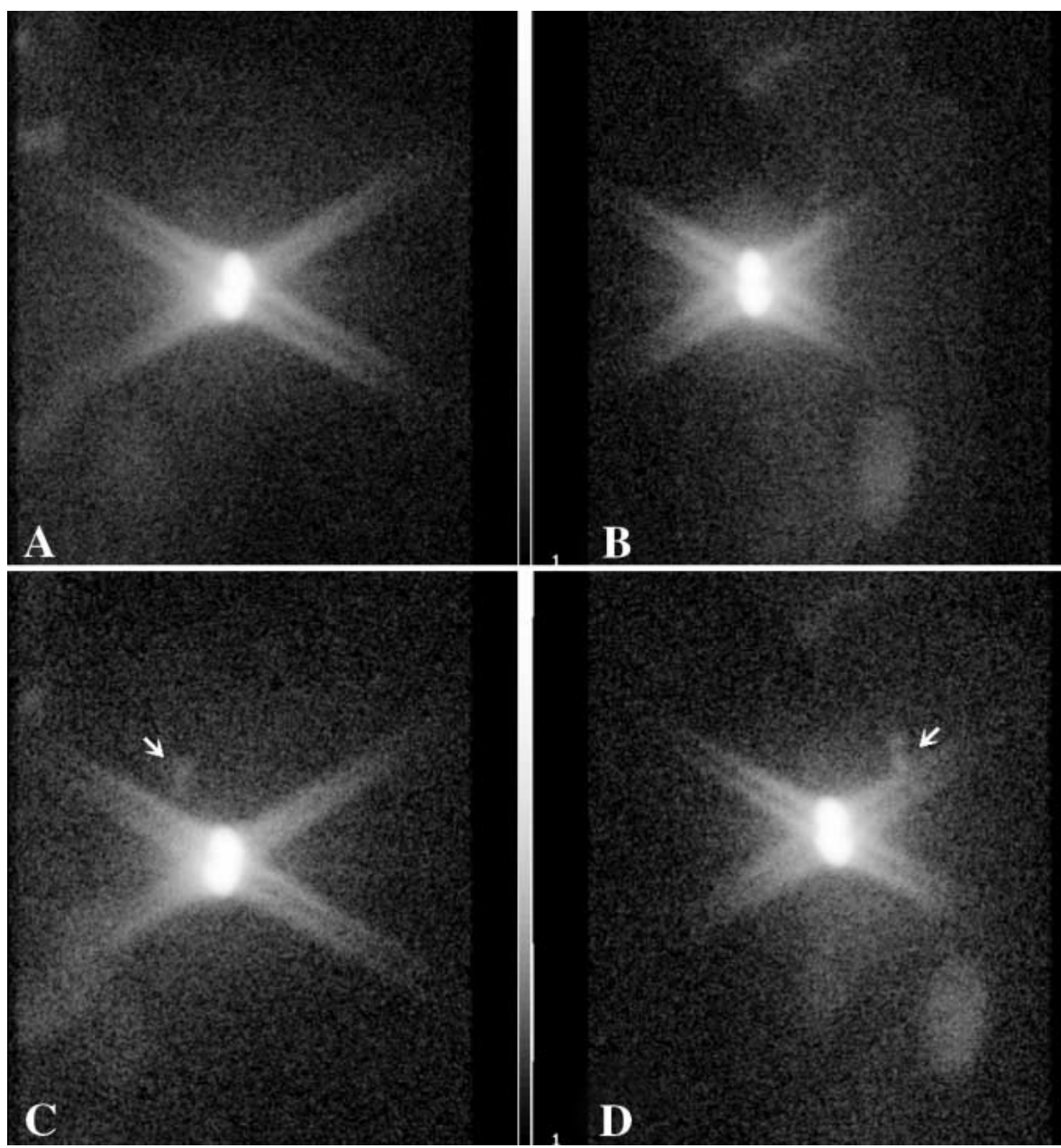

Table 3. Success rate for detection of the SN (isotopic/patent blue)

\begin{tabular}{lll}
\hline SN localization & Group A & Group B \\
\hline Isotopic method (\%) & $55 / 68(81 \%)$ & $21 / 27(78 \%)$ \\
Patent blue method (\%) & $36 / 68(53 \%)$ & $16 / 27(59 \%)$ \\
Both methods combined (\%) & $62 / 68(91 \%)$ & $23 / 27(85 \%)$ \\
\hline
\end{tabular}

Table 4. Comparison of the success rate for SN localization by LS and surgery

\begin{tabular}{lccc}
\hline & Positive LS & Negative LS & Total \\
\hline Positive surgery & 81 & 26 & 107 \\
Negative surgery & 8 & 3 & 11 \\
\hline Total & 89 & 29 & 118 \\
\hline
\end{tabular}

Lymphoscintigraphy: Evolution during the

Learning Curve was noted between lymph nodes detected by early images and SN detected on delayed images. However, SN visualization for the latter group was significantly improved on late images (from 55 to $71 \%, p<0.05$ ). Figure 1 shows an illustrative case in which the axillary SN was only visualized late both anteriorly and laterally. Changes in the identification of an SN focus of both early and late lymphoscintigraphic images indicated that the diagnostic value of late images decreased during the study (fig. 2). In fact, the negative rate for LS decreased by $10 \%$ during the study (fig. 3).

SN detection was comparable for the two groups with the isotopic method ( 81 and $78 \%, p=N S$ ) and also in the combined use of isotope and patent blue ( $91 \mathrm{vs.} 85 \%, \mathrm{p}=$ NS, table 3). The staining method was significantly less efficient, especially as compared to the combined use of isotope and patent blue dye (53 vs. $91 \%$ for group A and 59 vs. $84 \%$ for group $B, p<0.001)$. For the 118 patients in

Med Princ Pract 2003;12:17-22 


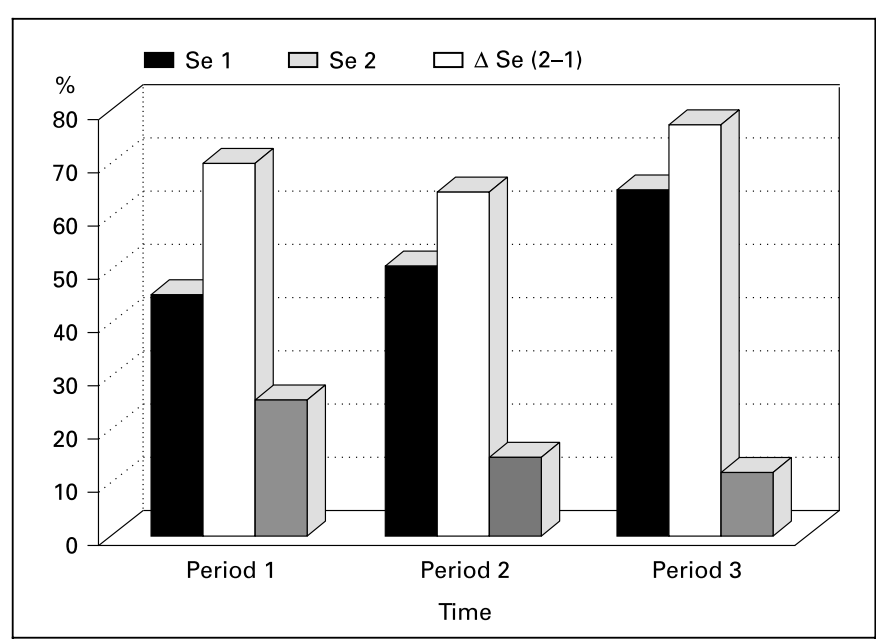

Fig. 2. Lymphoscintigraphic learning curves: changes in sensitivity. A period corresponds to a 6-month interval with 40 patients for each group. Se 1 = Identification of an SN focus on early images; Se 2 = identification of an $\mathrm{SN}$ focus on late images.

the study, the concordance between $\mathrm{SN}$ visualization by LS and intraoperative detection (isotope and/or patent blue) was $71.2 \%$ (table 4). Intraoperative detection localized SN in 24\% (26/107) of cases for which LS was negative. Surgery was negative in 11 cases, including 8 for which LS was positive.

\section{Discussion}

The search for $\mathrm{SN}$ in breast cancer is a recent and rapidly developing concept, which some authors consider to be the most significant therapeutic advance made within the last 10 years [10]. As outpatient surgery for this pathology has developed successfully in recent years [11], it would seem appropriate to adapt this detection technique to ambulatory conditions, if patients so desire.

Nanocis (Schering CIS Bio International), the tracer used in our study and the only one approved to date by the French drug administration, consists of rhenium sulfide colloids with a mean diameter of around $100 \mathrm{~nm}$. Labeling is easy to perform and is reproducible. The tracer was injected peritumorally at two sites. The best choice of anatomical site remains controversial, and the options are intratumoral, peritumoral, subareolar, subcutaneous or intradermal. Most authors consider that the intratumoral site is unreliable because of possible tracer sequestration. The other injection modes have all provided excellent results, and no truly comparative studies have been con-

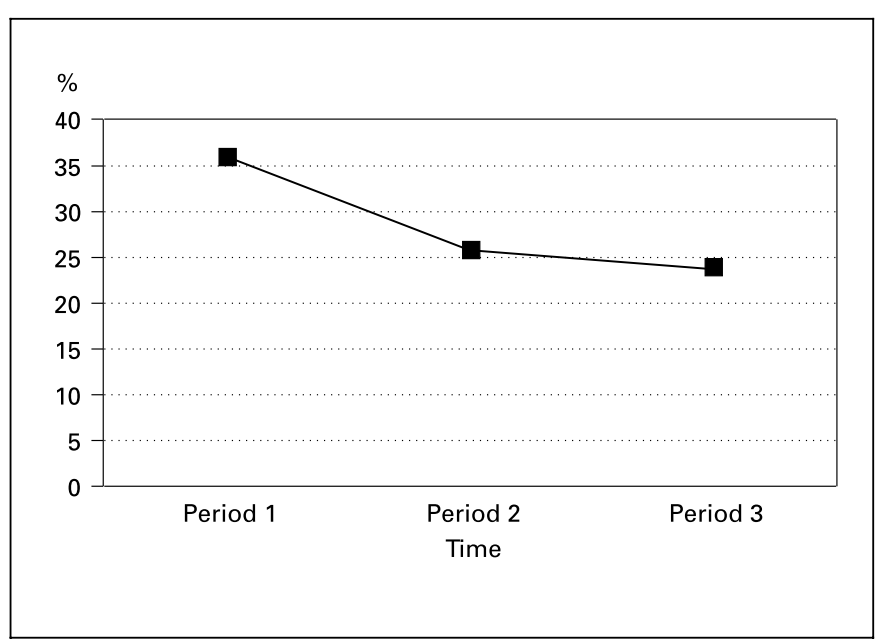

Fig. 3. Changes in negative detection rate in LS. A period corresponds to a 6-month interval.

ducted to show that one of these sites is preferable [1214].

A small amount of tracer was injected into two peritumoral sites. There are two schools of thought concerning the injection volume required. American teams recommend the injection of a large volume (4-6 ml) to dilate the lymph ducts and open up the drainage to the $\mathrm{SN}$. This choice increases of SN detected [10]. The activity of these nodes is then greater, which seems to facilitate intraoperative detection. European teams advocate the injection of a small volume $(0.1-04 \mathrm{ml})$ of a sufficiently concentrated product in association with light massage of the injection area. This procedure is considered to respect physiological conditions to best advantage [15]. As published results for the two techniques are excellent, the debate remains open.

There is less controversy in the literature concerning the activity injected. The studies generally recommend 15-30 MBq (range 1.8-370) of Nanocis, depending on the interval between injection and surgery [16]. Some authors [17] feel that two injections (one the day before surgery and the other just before the intraoperative detection) are necessary if detection is performed on the day after injection. Our group A received only a single injection, with administration of increased activity. The $\mathrm{SN}$ count rates in vivo indicated that the increased activity was sufficient, as the percentage of successful SN detection was the same for both groups. For group A, which received additional activity, the $\mathrm{SN}$ count rate tended to be higher than 
for group B (but the difference was not statistically significant). However, the confidence intervals overlapped considerably, which limited the statistical impact of this difference.

The proportion of positive LS $(\sim 70 \%)$ for both groups is in good agreement with the range in the literature (66$85 \%)$. No particular factors appear to account for the differences within this range [18].

The percentage of early positive LS differed significantly between the two groups, essentially because 'doubtful' results for group A were classified as negative prior to performance of the second series of images. This effect was particularly notable at the beginning of the study because of the learning phase required for interpretation of the images and the assurance that the patients would undergo a second imaging. The experience required, which parallels that necessary for the surgeon to obtain optimal results in intraoperative detection, involves around 20 patients [19,20]. An analysis of figures 2 and 3 confirms the importance of the learning process, as the benefit of a second series of images was reduced with time. Negative results with LS decreased by $10 \%$ between the beginning and end of the study.

In our series, the isotope-patent blue association gave the best results, which confirms the consensus in the literature that a combination of three approaches (preoperative LS, dye injection and intraoperative probe detection) is most efficient for localizing and isolating $\mathrm{SN}$ [21, 22]. The results for the staining method alone show an SN detection rate of $66-93 \%$. However, this method is not recommended for patients susceptible to atopy. The isotopic method alone is a more efficient method (a detection rate of 71-98\%). Techniques combining scintigraphy with intraoperative probe detection and the staining method give an SN detection rate of $83-100 \%$ and a negative detection rate of $0-15 \%$ [4]. In fact, false-negative results with the SN procedure correspond to patients with noninvasive $\mathrm{SN}$, but with histological involvement of the nodes removed by axillary resection. A combination of the staining technique with intraoperative probe detection gave a rate of $94 \%$ for $\mathrm{SN}$ and of around $0 \%$ for falsenegatives [5].

In view of the good results obtained with probe detection and the staining method, some authors feel that LS is unnecessary [23]. Moreover, in our study, surgical excision of the SN was achieved in $24 \%$ of cases for which LS was negative. However, in some cases scintigraphic assessment is useful for other reasons. First of all, it can provide information on different drainage routes (internal mammary, supraclavicular). In fact, a decreasing order of frequency has been determined for colloid transport by the external mammary system $(85 \%)$, the transpectoral route $(10 \%)$ and the internal mammary route $(5 \%)$ [24]. This information could be of importance for postsurgical (notably radiotherapeutic) treatments. Furthermore, a recent study [25] found that $26.8 \%$ (11/41) of internal mammary metastases were LS-positive in this location, including $7.3 \%(3 / 41)$ for which internal mammary invasion was not associated with axillary involvement. However, the clinical impact of these observations seems limited, given the low recurrence of the disease in the internal mammary lymph nodes [26]. Less successful identification of SN has been reported when LS was not performed (98\% with LS vs. $92 \%$ without) [23]. LS also provides more precise guidance for the surgeon, particularly for SN with a slightly atypical axillary topography (our of the typical axillary dissection area), allowing more rapid and reliable surgical exploration [27]. In a few cases, probe detection may fail to detect the SN despite LS positivity. In our study, the use of cutaneous landmarks for SN detected by LS might have helped account for the localization difficulties experienced by the surgeon. In the literature, these discordant cases have been attributed to the learning process for the surgical technique, especially for probe detection [20].

The recording of dynamic scintigraphic images at the time of injection is of definite value for localization of SN in melanoma. Indeed, the possibility, in melanoma, of an unforeseeable localization in a vast anatomical region requires images that are delocalized from the initial injection site. The usefulness of this procedure is less certain in breast cancer. Most authors deny any need for dynamic imaging [23] because the drainage area is more limited and quite predictable. However, the use of a large-field camera allows all possible drainage areas to be covered. The optimal interval between injection and imaging would appear to be $2 \mathrm{~h}$, as no significant increase in the number of positive LS was obtained beyond that time point [28]. In our study, the significant decrease in the diagnostic value of late images over time tends to corroborate this observation. However, a considerable scatter effect can persist during early LS and hinder image interpretation. Indeed, the SN may be very difficult to detect because it is situated along the path of the artefact. In this case, late images can allow improved interpretation. However, experience tends to minimize these difficulties. On the other hand, a shorter interval between injection and imaging $(<2 \mathrm{~h})$ increases the number of negative LS and limits the possibility of localizing extra-axillary drainage [29]. 


\section{Conclusion}

A learning phase is important not only for surgical detection of sentinel nodes in breast cancer, as emphasized in the literature, but also for the practice of LS in nuclear medicine. During the period in which experience is being acquired, two series of LS images tend to improve interpretation. Subsequently, a single image performed optimally $2 \mathrm{~h}$ after injection seems sufficient. LS, though considered unnecessary by certain authors and sometimes not performed, has various advantages, particularly in increasing the intraoperative detection rate significantly and in guiding the surgical act. The detection of $\mathrm{SN}$ in outpatient surgery requires an adaptation of the nuclear medicine facility to allow patients to benefit fully from the technique.

\section{References}

1 Veronesi U, Luini A, Galimberti V, Marchini S, Sacchini V, Rilke F: Extent of metastatic axillary involvement in 1,446 cases of breast cancer. Eur J Surg Oncol 1990;16:127-133.

2 Fisher B, Wolmark N, Redmond C, Deutsch M, Fisher ER: Findings from NSABP Protoco No. B-04: Comparison of radical mastectomy with alternative treatments. II. The clinical and biologic significance of medial-central breast cancers. Cancer 1981;48:1863-1872.

3 Cabanas RM: An approach for the treatment of penile carcinoma. Cancer 1977;39:456-466.

4 Morton DL, Wen DR, Wong JH, Economou JS, Cagle LA, Storm FK, Foshag LJ, Cochran AJ: Technical details of intraoperative lymphatic mapping for early stage melanoma. Arch Surg 1992;127:392-399.

5 Alex JC, Krag DN: Gamma probe guided localization of lymph nodes. Surg Oncol 1993;2: 137-143.

6 Giuliano AE, Kirgan DM, Guenther JM, Morton DL: Lymphatic mapping and sentinel lymphadenectomy for breast cancer. Ann Surg 1994;220:391-401.

7 Haigh PI, Hansen NM, Giulanio AE, Edwards GK, Ye W, Glass EC: Factors affecting sentinel node localization during preoperative breast lymphoscintigraphy. J Nucl Med 2000;41: 1682-1688.

8 Pijpers R, Meijer S, Hoekstra OS, Collet GJ, Comans EF, Boom RP, Van Diest PJ, Teule GJ: Impact of lymphoscintigraphy on sentinelnode identification with Technetium-99m-colloidal albumin in breast cancer. J Nucl Med 1997;38:366-368

9 Margolese RG, Lasry JC: Ambulatory surgery for breast cancer patients. Ann Surg Oncol 2000;7:181-187.

10 Krag D, Weaver D, Ashikaga T, Moffat F, Klimberg VS, Shriver C, Feldman S, Kusminsky R, Gadd M, Kuhn J, Harlow S, Beitsch P: The sentinel node in breast cancer: A multicenter validation study. N Engl J Med 1998; 339:941-946.
11 Dooley WC: Ambulatory breast cancer surgery. Ann Surg Oncol 2000; 7:174-175.

12 Veronesi U, Paganelli G, Galimberti V, Viale G, Zurrida S, Bedoni M, Costa A, De Cicco C, Geraghty JG, Luini A, Sacchini V, Veronesi P: Sentinel node biopsy to avoid axillary dissection in breast cancer with clinically negative lymph-nodes. Lancet 1997;349:1864-1867.

13 Borgstein PJ, Meijer S, Pijpers R: Intradermal blue dye to identify sentinel lymph node in breast cancer. Lancet 1997;349:1668-1669.

14 Krag DN, Harlow S, Weaver D, Ashikaga T: Radiolabeled sentinel node biopsy: Collaborative trial with the national cancer institute. World J Surg 2001;25:823-828.

15 De Cicco C, Cremonesi M, Luini A, Bartolomei M, Grana C, Prisco G, Galimberti V, Calza $\mathrm{P}$, Viale $\mathrm{G}$, Veronesi $\mathrm{U}$, Pagnelli G: Lymphoscintigraphy and radioguided biopsy of the sentinel axillary node in breast cancer. $\mathbf{J}$ Nucl Med 1998;39:2080-2084.

16 van der Ent FW, Kengen RA, van der Pol HA, Hoofwijk AG: Sentinel node biopsy in 70 unselected patients with breast cancer: Increased feasibility by using $10 \mathrm{mCi}$ radiocolloid in combination with blue dye tracer, Eur J Surg Oncol 1999;25:24-29.

17 Delpassand E, Hunt K, Ross M: Early and delayed breast lymphoscintigraphy using Tc99m filtered sulfur colloid. J Nucl Med 2000;5: 498P.

18 Borgstein PJ, Pijpers R, Comans EF, Van Diest PU, Boom RP, Meijer S: Sentinel lymph node in breast cancer: Guidelines and pitfalls of lymphoscintigraphy and gamma probe detection. J Am Coll Surg 1998;186:275-283.

19 Alazraki NP, Styblo T, Grant SF, Cohen C, Larsen T, Aarsvold JN: Sentinel node staging of early breast cancer using lymphoscintigraphy and the intraoperative gamma-detecting probe. Semin Nucl Med 2000;30:56-64.

20 Orr RK, Hoehn JL, Col NF: The learning curve for sentinel node biopsy in breast cancer: Practical considerations. Arch Surg 1999;134:764767.
21 Hill AD, Tran KN, Akhurst T, Yeung H, Yeh SD, Rosen PP, Borgen PI, Cody HS 3rd: Lessons learned from 500 cases of lymphatic mapping for breast cancer. Ann Surg 1999;229: 528-535.

22 Uren RF: Let every node count! J Nucl Med 2001;42:452-453.

23 Mc Masters KM, Wong SL, Tuttle TM, Carlson DJ, Brown CM, Dirk Noyes R, Glaser RL, Vennekotter DJ, Turk PS, Tate PS, Sardi A, Edwards MJ: Preoperative lymphoscintigraphy for breast cancer does not improve the ability to identify axillary sentinel lymph nodes. Ann Surg 2000;231:724-731.

24 Haigh PI, Hansen NM, Giuliano AE, Edwards GK, Ye W, Glass EC: Factors affecting sentinel node localization during preoperative breast lymphoscintigraphy. J Nucl Med 2000;41: 1682-1688.

25 van der Ent FW, Kengen RA, van der Pol HA, Povel JA, Stroeken HJ, Hoofwijk AG: Halsted revisited: Internal mammary sentinel lymph node biopsy in breast cancer. Ann Surg 2001; 234:79-84.

26 Pourquier D, Lemanski C, Farous P, Couty H, Delard R, Rouanet P, Dubois JB: Cancer of the breast: The return of lymphoscintigraphy? Bull Cancer 1998;85:675-684.

27 Peltier P, Guillard Y, Mey P, Drianno JC: Sentinel lymph node in 90 patients with breast cancer: Patent blue and radiodetection evaluation. Med Nucl 2000;24:207-216.

28 Valdes Olmos RA, Hoefnagel CA, Nieweg OE, Jansen L, Rutgers EJ, Borger J: Lymphoscintigraphy in oncology: A rediscovered challenge. Eur J Nucl Med 1999;26(suppl):S2-S10.

29 Yeung HWD, Cody HS III, Turlakow A, Riedel ER, Fey J, Gonen M, Nunez R, Yeh SD, Larson SM: Lymphoscintigraphy and sentinel node localization in breast cancer patients: A comparison between 1-day and 2-day protocols. Eur J Nucl Med 2001;42:420-423. 